\section{BMJ Open Respiratory Research}

\title{
Towards codesign in respiratory care: development of an implementation- ready intervention to improve guideline-adherent adult asthma care across primary and secondary care settings (The SENTINEL Project)
}

\author{
Lucia Crowther (D) , ${ }^{1}$ Mark Pearson, ${ }^{1}$ Helena Cummings, ${ }^{2}$ \\ Michael George Crooks (D) ${ }^{1,3}$
}

To cite: Crowther L, Pearson $\mathrm{M}$, Cummings $\mathrm{H}$, et al. Towards codesign in respiratory care: development of an implementation-ready intervention to improve guideline-adherent adult asthma care across primary and secondary care settings (The SENTINEL Project). BMJ Open Resp Res 2022;9:e001155. doi:10.1136/ bmjresp-2021-001155

- Additional supplemental material is published online only. To view, please visit the journal online (http://dx.doi. org/10.1136/bmjresp-2021001155).

Received 16 November 2021 Accepted 16 January 2022
Check for updates

(c) Author(s) (or their employer(s)) 2022. Re-use permitted under CC BY-NC. No commercial re-use. See rights and permissions. Published by BMJ.

For numbered affiliations see end of article.

Correspondence to

Lucia Crowther;

lucia.crowther@hyms.ac.uk

\section{ABSTRACT}

Short-acting beta agonist (SABA) overuse ( $\geq 3$ canisters annually) is associated with worse asthma outcomes and accounts for the majority of greenhouse gas emissions from asthma inhalers in England. Reducing SABA overuse aligns with the National Health Service long-term plan to optimise asthma treatment while minimising environmental impact, but adoption of local asthma guidelines for a SABA-free maintenance and reliever therapy strategy for step 3 asthma patients is limited. In this Perspective, we describe patient and staff involvement in a codesign process adapted from experience-based codesign (EBCD) principles to develop an implementationready intervention within a practice-relevant timescale. The codesigned intervention consists of five pillars: healthcare professional education; implementation of 'gold standard' prescribing practices; targeted asthma reviews; patient education and support; and real-time data monitoring and reporting of asthma care metrics. The codesign process contributed to all pillars and, by identifying potential individual and organisational barriers to implementation, enabled the development of plans to address these barriers.

In this Perspective, we reflect on the strengths and weaknesses of our codesign process, outline how EBCD principles can be used in respiratory research and propose actions for patients, health professionals, researchers and funders to develop the potential of EBCD in respiratory research.

\section{INTRODUCTION}

The involvement of healthcare professionals and patients is increasingly recognised as essential to the design of health services and interventions. ${ }^{1-3}$ However, codesign is rare in respiratory medicine. Our recent search of five major respiratory journals only identified two research articles which discussed how codesign or patient and public involvement had been conducted in the development of respiratory medicine interventions or plans for their implementation (online supplemental material 1). This suggests that little attention is paid to the involvement of healthcare professionals and patients, or that reporting of involvement is insufficient.

In this Perspective, we describe and critically reflect on our experience of involving patients and healthcare professionals in the design of an intervention to improve asthma care. Our intention is to open a discussion about the value and feasibility of codesign approaches in respiratory medicine, so that the processes can be developed, refined and applied to maximise codesign's role in improving the effectiveness and delivery of respiratory interventions. In this Perspective, we reflect on the strengths and weaknesses of our adaptation of the experience-based codesign (EBCD) process, outline how EBCD principles can be used in respiratory research and propose actions for patients, health professionals, researchers, and funders to develop the potential of EBCD in respiratory research.

Short-acting beta agonist (SABA) overuse ( $\geq 3$ canisters per year) is associated with worse asthma outcomes and accounts for the majority of greenhouse gas emissions from asthma inhalers in the UK. ${ }^{45}$ Reducing SABA overuse aligns with the National Health Service (NHS) long-term plan to optimise asthma treatment while minimising environmental impact, but adoption of local asthma guidelines for an SABA-free strategy using maintenance and reliever therapy for appropriate asthma patients is limited (online supplemental material 2). SABA rEductioN 
Through ImplemeNting Hull asthma guidELines (SENTINEL) aims to improve asthma outcomes and reduce the environmental impact of asthma medications in Hull and East Yorkshire by promoting implementation of the local adult asthma guideline.

Initial consultation was undertaken with key stakeholders including primary and secondary care clinicians and commissioners of asthma services. This consultation comprised a series of virtual meetings during which a multifaceted intervention was drafted. The draft intervention was circulated to meeting participants and refined in response to comments. This proposed intervention was then adapted and optimised through a series of codesign workshops with staff and patients from participating primary care networks (PCNs) to ensure it would meet the needs of the local population and healthcare providers, as described in this Perspective. The finalised intervention will be implemented across Hull over a 12-month period with robust evaluation and comprehensive evidence generation, with intervention delivery phased across PCNs in a step-wedge approach.

\section{How we adapted EBCD}

Recognising the importance of Involve's principles for co-producing research, ${ }^{6}$ which encompass the inclusion of all perspectives and skills, valuing of the knowledge of all stakeholders, and a reciprocal (rather than contractual) approach, we used a codesign process based on EBCD principles to develop and refine the SENTINEL intervention with patients and healthcare staff. ${ }^{7}$ Rather than seeking to 'perfect' processes or pathways from a service perspective, EBCD focuses on the experiences of patients as a vital source of knowledge about service redesign. ${ }^{8}$ Pivotal in this process are the facilitation of codesign workshops that bring together patients and staff, so that boundaries are crossed, new working relationships are formed, and new insights are obtained. ${ }^{9}$ Mindful of the limited contextual tailoring of many interventions in health services, ${ }^{10}$ we sought to use a codesign process adapted from EBCD principles, so that patients and staff could 'tell the untold stories' that would enable a contextually informed refinement of the SENTINEL intervention focused on the needs of the patients who could benefit from the intervention and the healthcare professionals with a role in delivering it.

EBCD processes have been honed to address the frequently encountered challenges posed by limited timescales, for example, in Locock et al s 'accelerated' process (seven stages with two pathways completed in 12 months), which was developed from the Point of Care Foundation's process (eight stages in 12 months). ${ }^{7} 1112$ We adapted Locock $e t a l$ s accelerated approach to build on the already-completed initial SENTINEL intervention development work and to enable the timely completion of codesign to inform implementation of the intervention in Hull and East Riding of Yorkshire PCNs (table 1).

Central to our adaptation of EBCD was close consideration of the views and experiences of healthcare staff as well as patients. There were both healthcare staff-facing (eg, the intervention pillars 'healthcare professional education' and 'real-time data monitoring and reporting of asthma care metrics') and patient-facing (eg, aspects of the 'targeted asthma reviews' and 'patient support and education') elements to the intervention. Healthcare staff were, therefore, 'recipients' or 'end-users' of the intervention as well as patients, and as such their insights were important for the development of the intervention and its implementation.

Although the reduced time frame of our codesign process necessitated a reduced number of stages and participants compared with previous studies, we preserved the focus on individuals' experiences and unique perspectives as the core element of our adapted approach. The themes that emerged during individual exploratory meetings were used as catalysts for discussion in group meetings to ensure individuals' insights

\section{Table 1 Comparison of the stages involved in different forms of experience-based co-design}

\section{EBCD Point of Care Foundation 2016}
1. Observe clinical areas
2. Interview staff, patients and families
3. Edit interviews into $25-30 \mathrm{~min}$ film of themed chapters
4. Staff feedback event
5. Hold patient feedback event (showing film to patients)
6. Hold joint patient-staff feedback event
7. Run co-design groups over 4-6 months
8. Hold a celebration event
1. Secondary analysis of narrative interviews from the HERG archive
2. Creation of trigger films
3. Discovery and engagement work with staff, including staff feedback event
4. Focus group workshop with local patients and carers
5. Co-design workshop with local staff, patients and carers
6. Co-design subgroups of staff, patients and carers
7. Final event

\section{AEBCD Locock et al.}

Adapted co-design process based on AEBCD (for use in the SENTINEL project)

1. Exploratory meetings by video link with clinical staff

2. Staff feedback event

3. Exploratory meetings over the phone with asthma patients

4. Co-design event 
remained central to the process of refining the intervention. By bringing healthcare professionals and patients together in joint discussion, we allowed debates to cross boundaries between different roles and for final decisions to draw from a wide base of experiences.

\section{METHODS}

Our adaptation of accelerated EBCD involved four key phases, each informed by Involve's principles for coproduction and with an emphasis on achieving Iedema $e t$ $a l$ s coproduction goals of enabling people to develop an appreciation of others' experiences and allow new ways of thinking to be opened up. ${ }^{69}$

1. Exploratory meetings by video call with clinical staff $(n=7)$

2. Staff feedback event $(n=1)$

3. Exploratory meetings by telephone with patients with asthma $(n=3)$

4. Codesign event $(n=1)$

A total of 15 participants were involved, representing asthma patients $(n=3)$, practice administrators $(n=1)$, advanced nurse practitioners $(n=2)$, respiratory nurse specialists $(n=1)$, practice nurses $(n=3)$, clinical pharmacists $(n=1)$, general practitioners $(n=3)$ and clinical research associates $(n=1)$.

\section{Recruitment}

Six GP practices across the target region were emailed invitations for staff to participate in the codesign process. Of these, two went on to have staff involved. The project lead recruited four asthma nurses working across two PCNs, who, in turn, recruited patient participants opportunistically during clinics.

All participants were offered reimbursement for their time, although not all accepted. Participants were sent plain English summaries of the project before participating, and before groups meeting agendas with topics outlined were circulated.

\section{Exploratory meetings (seven participants; 60 mins each)}

All participants were invited to an exploratory meeting with a researcher, in which the planned intervention components were discussed and feedback on potential challenges and solutions sought (table 2). A mixture of direct and open questions was posed, allowing novel topics to emerge. Defined as a service evaluation using the Health Research Authority's decision tool (http:// www.hra-decisiontools.org.uk/research/), the project did not require ethical approval.

Table 2 Topics and discussion points raised during exploratory meetings with clinical staff and asthma patients

\begin{tabular}{lll}
\hline $\begin{array}{l}\text { Topic from project proposal discussed } \\
\text { during meeting }\end{array}$ & $\begin{array}{l}\text { Discussion points on this topic for } \\
\text { meetings with clinical staff }\end{array}$ & $\begin{array}{l}\text { Discussion points on this topic for } \\
\text { meetings with asthma patients }\end{array}$
\end{tabular}

\begin{tabular}{lll}
\hline Healthcare Professional Education & Key topics that need to be \\
& addressed through education \\
& Preferences for how HCP education \\
& is delivered & n/a \\
Implementation of 'gold standard' & Current prescribing practices at the & participants' GP practice/PCN Participants' experiences of having \\
prescribing practice & Ease/difficulty of changing & asthma medication on repeat \\
& prescribing practices & Potential barriers to taking SABA \\
& inhalers off repeat prescription \\
& Key prescribing practices that \\
& should be encouraged in respect to \\
& asthma
\end{tabular}

Targeted asthma reviews

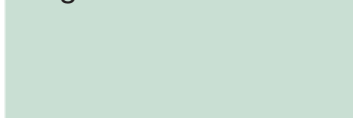

Patient support and education
- Preferences for how asthma reviews are conducted

- Potential barriers to implementing a focused period of asthma reviews

- Importance of patient support for guideline implementation

- Patient support for changing asthma medications
Preferences for how asthma reviews are conducted

- Encouraging non-attenders to attend asthma reviews

- Participants' use and understanding of asthma medications

- Perception of SABA inhalers

Patient support for changing asthma medications
Preferences for how asthma data is presented and which metrics should be available

- Preferences for how data is shared between practices and PCNs

HCP, Healthcare Professional; PCN, primary care network; SABA, short-acting beta agonist. 
Table 3 Topics used as the framework for group discussions during the staff feedback event, showing at which stage in the co-design process each topic was identified

\begin{tabular}{ll}
\hline Topic taken from project proposal & Topic identified during exploratory meetings \\
\hline Feedback on the three 'gold standard' prescribing practices & Booklet aid for staff to use during asthma reviews \\
Patient support for changing medication & Keeping standards of reviews consistent across the board \\
Preferences for how asthma data are presented & Encouraging non-attenders to attend asthma reviews \\
& The best way to inform and educate asthma patients about their \\
& medications \\
& Whether sharing data between practices could stigmatise those \\
& doing less well \\
\hline
\end{tabular}

\section{Staff feedback event (six participants; 120 min by video conference)}

This event aimed to collect group feedback on the challenges and solutions offered during exploratory meetings, and to address new topics not yet discussed with participants. Preplanned topics were used as catalysts for group discussions of the intervention (table 3).

By facilitating group discussions between heath care professionals, this event allowed participants to appreciate the perspectives of those working in different roles and to ultimately reach a consensus on the suggestions they made for the intervention's development.

\section{Codesign event (10 participants; 180 min by video conference)}

All participants were invited to attend the final codesign event. The aim was to finalise the intervention and agree next steps for implementation. Preplanned topics were used as catalysts for group discussions, with some carried forward from the staff feedback event and others having been identified during exploratory meetings (table 4).

Group discussions allowed participants to appreciate one another's perspectives and the project team to benefit from the participants' range of personal and professional experience. Discussions were constructive and wide ranging, with time given to consideration of novel ideas which arose. The ideas and suggestions raised throughout these conversations, along with the cumulative record of the previous meetings' feedback, ultimately led to several additions and modifications being made to the final specification of the intervention.

\section{COVID-19}

Due to COVID-19, all meetings were conducted over the phone or online. The pandemic appeared to impact the number of participants and event attendance: illness, having to self-isolate, childcare issues and unusually high workloads were all given as reasons for non-participation.

\section{REFINEMENT OF THE SENTINEL INTERVENTION THROUGH CODESIGN}

Before the codesign process began a project proposal was written in collaboration with key stakeholders in primary and secondary care outlining a draft five-component intervention. Throughout the codesign process, feedback was sought from participants on each of these components, with the final design of the intervention being agreed collaboratively.

The five intervention components were developed through co-design as follows:

Table 4 Topics used as the framework for group discussions during the co-design event, showing at which stage in the codesign process each topic was identified

\begin{tabular}{lll}
\hline $\begin{array}{l}\text { Topic identified during staff feedback } \\
\text { event }\end{array}$ & $\begin{array}{l}\text { Topic identified during exploratory meetings } \\
\text { with asthma patients }\end{array}$ & $\begin{array}{l}\text { Topic identified during exploratory meetings } \\
\text { with clinical staff }\end{array}$ \\
\hline $\begin{array}{l}\text { Feedback on the updated five 'gold } \\
\text { standard' prescribing practices }\end{array}$ & $\begin{array}{l}\text { Patients' views on using a stepped } \\
\text { approach to changing medications }\end{array}$ & $\begin{array}{l}\text { Whether the data from 2020 will be reliable } \\
\text { as a basis for comparison due to the effects } \\
\text { of the COVID-19 pandemic }\end{array}$ \\
How the 'gold standards' will work in & $\begin{array}{l}\text { Encouraging non-attenders to attend } \\
\text { practice as guidelines }\end{array}$ & Using Electronic Repeat Prescribing (eRD) \\
The proposed timeline for implementing & $\begin{array}{l}\text { Patient's preferences for how information is } \\
\text { for asthma medications }\end{array}$ \\
the 'gold standard' guidelines & presented and available to them & \\
Staff support for employing a stepped & \\
approach to changing a patient's & \\
medication & \\
Staff preferences for training resources & \\
How can staff training and development & \\
be a continuous process & \\
Preferences for how asthma data is \\
presented and shared between practices \\
and PCNs
\end{tabular}

$\mathrm{PCN}$, primary care network. 
Implementation of 'gold standard' prescribing practice

The 'gold standard' prescribing practices were significantly developed through the codesign process, taking them from three to five in number and refining the wording of each standard (online supplemental material 3). A clearer understanding of their acceptability to healthcare professionals and potential obstacles to their implementation were afforded through group discussions.

\section{Healthcare professional education}

The codesign process provided evidence for the acceptability of proposed ideas. The proposition that education be delivered through a range of facilitated sessions as well as via written and digital resources was unanimously supported by staff participants, providing confidence that this approach would be well-received by healthcare professionals.

\section{Targeted asthma reviews}

Although healthcare professionals agreed on principle with the importance of conducting a programme of targeted reviews, during exploratory meetings, most healthcare professionals raised concerns about the additional workload required to complete these reviews within the 2-month time period. Nurses also raised concerns about the repetitive nature of conducting numerous reviews in a day, arguing the quality of reviews would drop as staff became tired. To address this, the involvement of the NHS business partner, Interface Clinical Services, was formalised in the final intervention specification to support practice staff to complete asthma reviews.

Additionally, to reduce the number of reviews required in the first 2 months of implementation, it was agreed during the final codesign event that high SABA users (prescribed 6+SABA inhalers annually) would initially be targeted for asthma reviews, with lower SABA users invited for review throughout the following 12 months. This would spread workload more manageably and stagger follow-up reviews.

\section{Patient support and education}

Patients and healthcare professionals agreed that patient support and education would need to be a central focus of the project to make the intervention successful. It was stressed that many patients lack basic knowledge about asthma and its management, so education should not shy away from teaching basic principles. Providing healthcare professionals with a library of resources to aid them in supporting patients during asthma reviews was seen as essential.

Suggestions for specific areas of need for patient support and education were made by both patients and healthcare professionals, and these were incorporated into the plans for staff training materials to ensure healthcare professionals would be confident delivering appropriate patient support.

\section{Real-time data monitoring and reporting of asthma care metrics}

The discussions around the data monitoring and reporting of asthma care metrics led to 'the number of inhaler technique reviews' being added to the metrics potentially being reported in the new system. Concern over whether sharing data between practices and PCNs could stigmatise underperforming practices was a novel theme introduced by healthcare professionals during the staff feedback event, and allowed the reporting system to be more sensitively designed.

Healthcare professionals' preferences for reporting asthma metrics were overwhelmingly for an opt-in system, which would allow practices to share their data with others within their PCN and see each other's figures, allowing 'friendly competition' while encouraging the possibility of offering support and advice to colleagues in underperforming practices.

Healthcare professionals also raised concerns that year-onyear comparisons will be skewed for 2020 due to prescription changes during the pandemic, and this will be taken into account for data collection and analysis.

\section{Other findings of the codesign process}

In addition to the development of the five components of the intervention, the co-design process also highlighted other issues to be considered during implementation:

- Implementing the intervention will be different at practices across the region due to differences in patient demographics, widely varying prescribing processes at different practices, and the different ways practices are run internally.

- Change will take time and require a continual process of support rather than a one-off approach to training and education.

The first point will be taken into account during the evaluation of the intervention's effectiveness. The second point is difficult to plan for in the initial stage of the implementation as continued support will ultimately need to be provided internally within practices. However, motivating and encouraging staff in the initial stages could help keep up momentum for the changes being made past the initial 12 months of roll out.

\section{DISCUSSION}

The codesigned SENTINEL intervention introduces evidence-based changes in practice that represents a significant shift from SABA inhaler prescription and use. Approaching intervention design through a process of codesign with healthcare professionals and patients allowed us to explore ways to make changes feel grassroots rather than being imposed onto 
individuals as well as developing plans for making the changes sustainable and long-term.

Due to the restricted time line for our adaptation of EBCD, participant recruitment was limited and, in particular, included relatively few patients. Although we benefited from a broad range of clinical participants from a variety of roles, this arguably led to the codesign results being more focused towards the viewpoint of healthcare professionals. The lower number of patient participants resulted in the cancellation of a separate patient-only feedback event, which was initially intended for the codesign process. Despite this, patient participants raised valuable points, providing a much clearer understanding of patient hesitancy around changes to SABA prescriptions and allowing the appropriate and sensitive design of patient support materials. They were actively involved in discussions during the final codesign event, providing an invaluable balance to healthcare professionals' perspectives.

After the final codesign event several participants (both patients and healthcare professionals) spontaneously sent feedback to the researcher to say they had enjoyed being involved in the codesign process and had found it positive, encouraging and interesting. One healthcare professional wrote, 'I think working in partnership in this way is definitely the way forward and will lead to the best outcomes'.

\section{CONCLUSION}

It has been possible to deliver a codesign process adapted from accelerated EBCD principles under the exceptional conditions of the 2020 COVID-19 pandemic, consistent with Involve's coproduction principles and with demonstrable refinements to the SENTINEL intervention that are likely to improve its implementation and impact. While the number of codesign participants was modest $(n=15)$, this was not atypical for codesign processes that emphasise depth and quality of participation over scale, and stakeholder diversity was well represented with regards to professional roles. Our intention is not to present our adapted codesign process as a 'best practice' model for EBCD; rather we have critically reflected on our experience of using EBCD principles in order to open a discussion about codesign in respiratory care and have described in this Perspective how patient and healthcare professional involvement in codesign played a fundamental role in the development and strengthening of the SENTINEL intervention. On the basis of this experience and the contributions of codesign in other areas of healthcare, we strongly encourage funders to ensure sufficient resources for codesign in respiratory research, for journals to allow sufficient space for reporting codesign activities, and for respiratory research centres to support the development of researchers', healthcare professionals' and patients' skills in codesign. This will enable the perspectives and experience of patients and healthcare professionals to shape the interventions which they will benefit from and implement.

The effectiveness of the SENTINEL intervention on asthma outcomes of adults in six PCNs in Hull and the East Riding of Yorkshire will be evaluated using anonymised, routinely collected patient data. During implementation of the intervention throughout 2021, feedback will be collected from participating healthcare professionals in order to develop an implementation strategy for optimal delivery of the intervention, bringing forward the importance of consultation with end users into the rollout and evaluation of the intervention.

\section{Author affiliations}

${ }^{1}$ Hull York Medical School, Institute of Clinical and Applied Health Research, University of Hull, Hull, UK

${ }^{2}$ Respiratory Medicine, Holderness Health Primary Care Network, Hull, UK ${ }^{3}$ Academic Respiratory Medicine, Hull University Teaching Hospitals NHS Trust, Hull, UK

Acknowledgements Our thanks to all of the patients and health carehealthcare professionals who took the time to make such valuable contributions to the co-design process.

Contributors MGC and HC conceptualised the SENTINEL Project, with LC, MP and MGC conceptualising the co-design process. LC, MP and MGC developed and facilitated the co-design workshops. LC and MP wrote the first draft of the manuscript, with $\mathrm{HC}$ and MGC making critical contributions and refinements. All authors read and approved the final manuscript.

Funding This work was funded by a grant from AstraZeneca UK. Grant/award number: not applicable.

Competing interests LC and MP: none declared. HC has received honoraria from AstraZeneca, Chiesi and Pfizer. MGC has received honoraria and/or non-financial support from AstraZeneca, BI, Chiesi, GSK, Novartis and Pfizer. MGC has received grants from AstraZeneca, Bl, Chiesi and Pfizer.

\section{Patient consent for publication Not applicable.}

Ethics approval This study involves human participants but was not approved by Defined as a service evaluation using the Health Research Authority's decision tool (http://www.hra-decisiontools.org.uk/research/), the project did not require ethical approval. Participants gave informed consent to participate in the study before taking part.

Provenance and peer review Not commissioned; externally peer reviewed.

All data relevant to the study are included in the article or uploaded as supplementary information.

Supplemental material This content has been supplied by the author(s). It has not been vetted by BMJ Publishing Group Limited (BMJ) and may not have been peer-reviewed. Any opinions or recommendations discussed are solely those of the author(s) and are not endorsed by BMJ. BMJ disclaims all liability and responsibility arising from any reliance placed on the content. Where the content includes any translated material, BMJ does not warrant the accuracy and reliability of the translations (including but not limited to local regulations, clinical guidelines, terminology, drug names and drug dosages), and is not responsible for any error and/or omissions arising from translation and adaptation or otherwise.

Open access This is an open access article distributed in accordance with the Creative Commons Attribution Non Commercial (CC BY-NC 4.0) license, which permits others to distribute, remix, adapt, build upon this work non-commercially, and license their derivative works on different terms, provided the original work is properly cited, appropriate credit is given, any changes made indicated, and the use is non-commercial. See: http://creativecommons.org/licenses/by-nc/4.0/.

\section{ORCID iDs}

Lucia Crowther http://orcid.org/0000-0003-3706-6073

Michael George Crooks http://orcid.org/0000-0001-6876-0258 


\section{REFERENCES}

1 Yardley L, Morrison L, Bradbury K, et al. The person-based approach to intervention development: application to digital health-related behavior change interventions. J Med Internet Res 2015;17:e30.

2 Gray-Burrows KA, Willis TA, Foy R, et al. Role of patient and public involvement in implementation research: a consensus study. BMJ Qual Saf 2018:27:858-64.

3 Locock L, Boaz A. Drawing straight lines along blurred boundaries: qualitative research, patient and public involvement in medical research, co-production and co-design. Evidence \& Policy 2019:15:1-13.

4 Janson C, Henderson R, Löfdahl M, et al. Carbon footprint impact of the choice of inhalers for asthma and COPD. Thorax 2020;75:82-4

5 Bloom Cl, Cabrera C, Arnetorp S, et al. Asthma-Related Health Outcomes Associated with Short-Acting $\beta_{2}$-Agonist Inhaler Use: An Observational UK Study as Part of the SABINA Global Program. Adv Ther 2020;37:4190-208.

6 INVOLVE. Guidance on co-producing a research project. Southampton: INVOLVE, 2018.
7 Donetto S, Tsianakas V, Robert G. Using experience-based Codesign to improve the quality of healthcare: mapping where we are now and establishing future directions. London: King's College London, 2014

8 Bate P, Robert G. Experience-based design: from redesigning the system around the patient to co-designing services with the patient. Qual Saf Health Care 2006;15:307-10.

9 ledema R, Merrick E, Piper D, et al. Codesigning as a Discursive practice in emergency health services: the architecture of deliberation. J Appl Behav Sci 2010;46:73-91.

10 Davis MM, Howk S, Spurlock M, et al. A qualitative study of clinic and community member perspectives on intervention toolkits: "Unless the toolkit is used it won't help solve the problem". BMC Health Serv Res 2017;17:497.

11 Locock L, Robert G, Boaz A. Testing accelerated experience-based co-design: a qualitative study of using a national archive of patient experience narrative interviews to promote rapid patient-centred service improvement. health services and delivery research. Southampton (UK): NIHR Journals Library, 2014.

12 Point of Care Foundation. EBCD: Experience-based co-design toolkit, 2016. Available: https://www.pointofcarefoundation.org.uk/ resource/experience-based-co-design-ebcd-toolkit/ [Accessed Dec 2020]. 